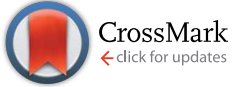

Cite this: DOI: 10.1039/c4sm01972e

\title{
Relating foam and interfacial rheological properties of $\beta$-lactoglobulin solutions
}

\author{
M. Lexis and N. Willenbacher*
}

We have determined bulk rheology of $\beta$-lactoglobulin (BLG) foams and surface viscoelasticity of corresponding protein solutions by varying $\mathrm{pH}$ as well as type, valency and concentration of the added salt in a wide range. Foam rheology was characterized by the storage modulus $G_{0}$, the apparent yield stress $\tau_{y}$, and the critical strain $\gamma_{c \text {,foam }}$ defining the cessation of the linear viscoelastic response. These quantities were determined at gas volume fractions $\phi$ between $82 \%$ and $96 \%$. Surface viscoelasticity was characterized in shear and dilation, corresponding shear and dilational moduli $G_{i}^{\prime}, E^{\prime}$ as well as the critical stress $\tau_{c \text {,surface }}$ and strain $\gamma_{c \text {,surface }}$ marking the onset of non-linear response in oscillatory surface shear experiments were determined at fixed frequency. Beyond the widely accepted assumption that $G_{0}$ and $\tau_{y}$ are solely determined by the Laplace pressure within the droplets and the gas volume fraction we have found that both quantities strongly depend on corresponding interfacial properties. $G_{0}$ increases linearly with $G_{i}^{\prime}$ and even stronger with $E^{\prime}, \tau_{y}$ varies proportional to $\tau_{c, \text { surface }}$ and $\gamma_{c \text {,foam }}$ scales linearly with $\gamma_{c, \text { surface }}$. Furthermore, deviations from these simple scaling laws with significantly higher reduced $G_{0}$ and $\tau_{y}$ values are observed only for foams at $\mathrm{pH} 5$ and when a trivalent salt was added. Then also the dependence of these quantities on $\phi$ is unusually weak and we attribute these findings to protein aggregation and structure formation across the lamellae than the dominating bulk rheology.

Received 3rd September 2014 Accepted 6th October 2014

DOI: $10.1039 / c 4 s m 01972 e$

www.rsc.org/softmatter storage modulus and yield stress initially proposed by Mason et al. and extended in our previous study are shown.

$$
\begin{gathered}
G_{0}=a\left(\frac{\sigma}{r_{32}}\right) \phi\left(\phi-\phi_{\mathrm{c}}\right) \\
\tau_{\mathrm{y}}=k\left(\frac{\sigma}{r_{32}}\right)\left(\frac{\eta_{\mathrm{L}}}{\eta_{\mathrm{W}}}\right)^{0.3}\left(\phi-\phi_{\mathrm{c}}\right)^{2}
\end{gathered}
$$

where $\sigma$ is the surface tension, $r_{32}$ the Sauter mean radius, $\phi$ the gas volume fraction, and $\phi_{c}$ represents the maximum packing fraction of the bubbles before they start to deform into nonspherical shapes. The latter has usually been an estimated value but can also be calculated from the measured bubble size distribution, as we have proposed recently. ${ }^{7}$ The prediction of the yield stress includes an empirically determined factor for the (weak) contribution of the liquid viscosity, where $\eta_{\mathrm{L}}$ is the continuous phase viscosity and $\eta_{\mathrm{w}}$ the water viscosity under the same conditions. This phenomenological extension of the model equation proposed by Mason et al. ${ }^{\mathbf{1 , 2}}$ has been derived from measurements on foams made from casein, whey protein isolate and a mixture of synthetic surfactants. The solvent viscosity was varied using different water-glycerol mixtures and sugar solutions. ${ }^{8}$ Each equation includes a numerical prefactor, $k$ and $a$, respectively. Values between 0.5 and 1 have been used for these constants so far in the literature without further discussion. ${ }^{1,2,5}$ In a recent study we found $k$ values between 1 and 7 and $a$ values varying between 2 and 22 . 
The film dilational modulus is defined by $E=-A \mathrm{~d} \pi / \mathrm{d} \ln A$ where $\pi$ is the surface pressure, and $A$ is the area of the film. The modulus $E$ is thus a measure of the resistance of a film to change in its area. The surface shear rheology gives information about the resistance of an adsorbed layer at the interface against shear. Hence, it is sensitive to the structural state of the adsorbed molecules. From a colloidal view a surface elastic modulus arises either from attractive interactions between neighboring particles or particles caged at high packing density. ${ }^{9}$ From interfacial oscillatory shear measurements another quantity can be extracted, the critical shear stress $\tau_{\mathrm{c} \text {,surface }}$ which denotes the end of the linear viscoelastic regime. For $\tau>\tau_{\mathrm{c} \text {,surface }}$ the deformation response becomes non-linear indicating a structural change in the surface layer. ${ }^{\mathbf{1 0}}$ Surface shear rotational experiments also show yielding behavior for several protein solutions which was investigated by Martin et al. ${ }^{\mathbf{1 1}}$ They came to the conclusion that the critical shear stress indeed induces a fracture within the protein film and can therefore be regarded as an intrinsic property of the protein layers. A higher critical stress represents a higher strength of the protein layer.

Surface rheology has been mainly discussed in terms of foam formation and stability. ${ }^{\mathbf{1 2 - 1 6}}$ Little is known so far about the correlation between foam rheology and the surface viscoelasticity of corresponding protein solutions although these features must be coupled since shearing a foam induces stretching and compression of the lamellae and hence the surfactant layer at the air liquid interface. The group of Cohen-Addad has thoroughly investigated the linear viscoelastic response $G^{*}$ of surfactant foams in a broad frequency range. Based on the model of Princen ${ }^{\mathbf{1 7}}$ they have proposed a relationship between $G^{*}(\omega)$ and the complex angular modulus $A^{*}(\omega)$ determined from dynamic compression tests of two adjacent bubbles connected by a single lamella. The quantity $A^{*}(\omega)$ is further assumed to be proportional to the dilational modulus $E^{*}=E^{\prime}+i E^{\prime \prime}$. In particular, they could show that the fast relaxation processes observed in foams are determined by the surfactant transport within the liquid films. ${ }^{18}$ The frequency $\omega_{\mathrm{c}}$ characterizing the onset of this scaling regime is assumed to be proportional to the ratio of the dilational modulus $E^{\prime}$ and the effective interfacial viscosity including the surface viscosity $E^{\prime \prime} / \omega_{\mathrm{c}}$, the solution viscosity and the lamellar thickness as well as the bubble diameter. Different scaling laws relating $\omega_{\mathrm{c}}$ to the foam modulus $G$ are proposed for rigid and mobile interfaces and these scaling laws are confirmed experimentally for two different types of surfactant foams. ${ }^{19}$ Deviations from the simple $G^{*} \sim \omega^{1 / 2}$ scaling are observed for foams made from surfactants providing very rigid interfaces $\left(E^{*} \approx 102 \mathrm{~Pa}\right) \cdot{ }^{20}$ So far, a systematic comparison between foam plateau modulus and interfacial shear or dilational moduli for protein foams is missing.

The viscous stress in continuously sheared foams scales with the capillary number $\mathrm{Ca}$ as $\tau_{\mathrm{v}} \sim \mathrm{Ca}^{n}$ and the exponent $n$ depends on surface mobility and viscoelasticity. For rigid interfaces $n=$ $1 / 4$ and for mobile interfaces $n=1 / 2$ have been predicted theoretically and confirmed experimentally. ${ }^{21-23}$
A direct empirical correlation between the yield stress and the interfacial dilation modulus $E^{\prime}$ of whey protein foams made at different $\mathrm{pH}$, concentration and valency of added salt has been proposed by Davis et al. ${ }^{24}$ However, they did not take into account the effect of bubble size (distribution) and gas volume fraction on $\tau_{\mathrm{y}}$, although $\mathrm{pH}$ and ionic strength are known to affect the absolute value of this quantity substantially. Dimitrova and Leal-Calderon ${ }^{25}$ reported a correlation between shear modulus of concentrated emulsions stabilized by different proteins and dilational moduli of the corresponding protein solutions. But it should be noted that their $E^{\prime}$ values were taken from the literature probably determined at protein concentration, $\mathrm{pH}$ and ionic strength conditions different from those relevant for the probed emulsions. Finally, it should be mentioned that also for particle stabilized so-called Pickering emulsions the modulus $G_{0}$ is not only determined by the interfacial tension between the liquid phases but also by an elastic contribution resulting from the attractive interaction among the stabilizing particles. ${ }^{26}$

In this study we attempt to directly correlate the surface and foam rheological properties of $\beta$-lactoglobulin (BLG) solutions. Therefore, the interfacial layer properties were systematically changed by varying the ionic strength, the type of salt and the solution $\mathrm{pH}$. We propose a unique relationship between foam modulus $G_{0}$ and interfacial moduli $G_{\mathrm{i}}^{\prime}$ or $E^{\prime}$, between the foam yield stress $\tau_{\mathrm{y}}$ and the critical stress or strain at which an interfacial layer structure breaks down. We demonstrate the validity of this correlation in a wide range of gas volume fractions irrespective of bubble size distribution and Laplace pressure. Finally we discuss the limitation of this approach in terms of structure formation across lamellae induced under certain conditions of ionic strength, ion valency and $\mathrm{pH}$.

\section{Experimental details}

\subsection{Solution preparation and measurements}

Solutions of $1 \mathrm{wt} \% \beta$-lactoglobulin (BLG, used as received) were prepared by dissolving the protein powder kindly provided by the group of Ulrich Kulozik (University of Munich, Germany) in ultrapure water (Millipore, $18 \mathrm{M} \Omega$ ). Variation of $\mathrm{pH}$ was achieved by adding appropriate amounts of $\mathrm{NaOH}$ or $\mathrm{HCl}$ (Carl Roth $1 \mathrm{~N}$ standard solutions), respectively. Ionic strength was varied by adding $\mathrm{NaCl}$ (99.5\%, Roth Chemicals) between 10 and $100 \mathrm{mM}$. The influence of ion type and valency was investigated by the addition of $50 \mathrm{mM} \mathrm{KCl,} \mathrm{LiCl}, \mathrm{NH}_{4} \mathrm{Cl}(99 \%$, Roth Chemicals), $\mathrm{CaCl}_{2}$ (98\%, Roth Chemicals) or $\mathrm{NdCl}_{3}$ (99.9\%, Alfa Aesar).

The surface tension of all solutions was measured by the pendant drop method (Krüss, DSA 100) at $21^{\circ} \mathrm{C}$ and a drop age of $30 \mathrm{~min}$ as described elsewhere. ${ }^{7}$

The liquid viscosities were measured with an Ares rotational rheometer (TA Instruments) using Couette geometry (17/16.5 $\mathrm{mm}$ ). All solutions showed Newtonian behavior in the range of imposed shear rates $\dot{\gamma}=10-1000 \mathrm{~s}^{-1}$ with viscosities between 0.94 and 1.1 mPas.

Interfacial dilational elasticities were determined at $21{ }^{\circ} \mathrm{C}$ and a drop age of 30 min using the oscillating bubble method 
(Krüss, DSA 100). The oscillations were generated by using a piezo pump that pulsed with a frequency of $0.1 \mathrm{~Hz}$ and amplitude of 0.3 . The amplitude resulted in drop deformation between 2 and $3 \%$, depending on the drop volume. As the drop was generated manually it was not possible to keep the volume for every measurement exactly the same. Oscillatory deformation was applied for a time period of $100 \mathrm{~s}$ and 1200 pictures were analyzed to calculate $E^{*}=E^{\prime}+i E^{\prime \prime}$.

Interfacial shear viscoelastic properties were determined at $25{ }^{\circ} \mathrm{C}$ and a surface age of $30 \mathrm{~min}$ with a stress controlled rotational rheometer (TA Instruments, DHR3) using the double wall ring geometry $\left(D_{\text {ring }}=70 \mathrm{~mm}\right)$. Details about this measuring geometry can be found in. ${ }^{27}$ The viscoelastic properties were recorded at a frequency of $0.7 \mathrm{~Hz}$ and a deformation amplitude of $1 \%$, which did not exceed the linear viscoelastic regime of any sample solution. From these measurements we have determined the surface elastic modulus $G_{\mathrm{i}}^{\prime}$. Measurements with increasing deformation amplitude allowed for the determination of the width of linear viscoelastic regime (LVE). When the non-linear deformation response sets in, $G_{\mathrm{i}}^{\prime}$ starts to decrease and the end of the LVE was determined to reach when $G_{\mathrm{i}}^{\prime}=0.9^{*} G_{\mathrm{iLVE}}^{\prime}$. At this point the critical deformation $\gamma_{\mathrm{c} \text {,surface }}$ and shear stress $\tau_{\mathrm{c} \text {,surface }}$ were extracted.

It should be noted that we have characterized the interfacial rheology of protein solutions at the same concentration at which foam preparation and foam rheology was done. This is in contrast to many other studies on interfacial rheology where experiments were done at much lower concentration of amphiphiles in order to ensure a monolayer of the surface active ingredient at the air/water interface. But one has to be aware that the structure of an interfacial layer, especially in the case of proteins, can substantially change with concentration and a correlation of foam and interfacial rheology can only be expected if the interfacial layer is the same in both sets of experiments. However, multiple layers of proteins may be present at the surface, i.e. the thickness of the layer may not be negligible. Therefore, the measured quantities $E^{\prime}, G_{\mathrm{i}}^{\prime}$ have to be treated as apparent values.

\subsection{Foam preparation and measurements}

The protein solutions were preheated to $50{ }^{\circ} \mathrm{C}$ in a water bath to obtain foams that are stable enough for reproducible rheological measurements. The increase in temperature speeds up adsorption kinetics but does not affect the protein structure. Foams were produced using a glass filter fused in a glass pipe as described elsewhere ${ }^{7}$ and nitrogen was purged through the pores at $\dot{V}=60 \mathrm{ml} \mathrm{min}{ }^{-1}$. As soon as the foam reached the column height the nitrogen flow was stopped and recording of the foam age was started.

The time-dependent gas volume fraction was determined using a conductivity electrode with an integrated temperature sensor (WTW, Cond 340i) as described in a previous study. ${ }^{7}$ The measuring gap of the electrode had a length of $2 \mathrm{~cm}$. The foam volume within the gap is similar to that we have used for rheological characterization. Hence, the measured conductivity is an appropriate average value. Conductivity measurements were performed in a region of the foam column close to that from where the samples for rheological measurements were taken.

Fig. 1 shows the drainage velocity for the protein foams with different ionic strengths and different $\mathrm{pH}$. Increasing the amount of $\mathrm{NaCl}$ leads to slower liquid drainage. This can be attributed to the reduced electrostatic repulsion between the proteins leading to closer protein packing and increased probability of aggregation. Such protein aggregates are able to plug the junctions of the Plateau borders so that the drainage of the liquid is slowed down. Moreover, the surface mobility can have an influence on foam drainage. ${ }^{28,29}$ As the interface is supposed to become more rigid with increasing ionic strength (at least upon addition of $20 \mathrm{mM}$ as discussed in Section 3.1.4) this might be an additional reason for the slower drainage. The $\mathrm{pH}$ dependent drainage velocity shows a minimum at $\mathrm{pH}$. This is very close to the isoelectric point (IEP) where the protein net charge is close to zero and therefore, aggregates plugging the liquid channels occur more frequently. Higher distance to the IEP increases the protein solubility and at the same time the drainage velocity.

The bubble size distribution was determined by taking pictures with an endoscopic CCD camera (Lumenera LU 160, resolution $1392 \times 1040$ ) that was placed inside the foam. The Sauter mean radius $r_{32}$ was extracted from image analysis using the software iPS (Visiometrics, Germany).

Foam rheological measurements were carried out with Rheoscope 1 (Thermofisher, Germany) using parallel plate geometry with a diameter of $60 \mathrm{~mm}$. The surfaces were covered with sandpaper to minimize wall slip effects and the gap was set to $6 \mathrm{~mm}$. The measurement time was $60 \mathrm{~s}$ in order to limit time dependent changes in the foam structure. Each foaming system was measured at different foam ages and hence, different gas volume fractions $\phi$ between 82 and $94 \%$.

The apparent yield stress was determined from steady shear measurements where increasing stresses were applied. Depending on the foam composition the initial stresses were set between 3 and $5 \mathrm{~Pa}$ and the final stresses between 50 and 125 Pa. As already extensively discussed in ref. 7 the apparent yield stress is independent of the start and end point of the stress ramp experiment as well as on the number of data points taken. In particular, it was shown that the sample deformation within
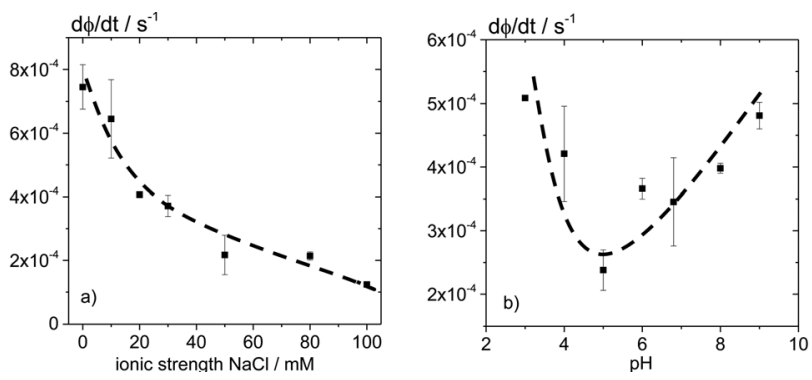

Fig. 1 Drainage velocity of the protein foams with different ionic strengths (a) or $\mathrm{pH}$ (b). Data were calculated from the time period in which each foam drained from $85 \%$ to $86 \%$ gas volume fraction. 
the gap is non-uniform when the yield point is exceeded. We have assumed that this effect is weak just around the yield point, and experiments that determined $\tau_{\mathrm{y}}$ and $\gamma_{\mathrm{c}}$ were highly reproducible but the parameters should be treated as apparent values.

The moduli $G^{\prime}$ and $G^{\prime \prime}$ of the foam were determined from oscillatory shear measurements by varying the stress amplitude at a frequency $f=1 \mathrm{~Hz}$. The moduli did not show frequency dependence between 0.01 and $10 \mathrm{~Hz}$. Hence, the measured $G^{\prime}$ value in the linear viscoelastic regime is called plateau modulus $G_{0}$. The deformation amplitude that decreased $G^{\prime}$ down to

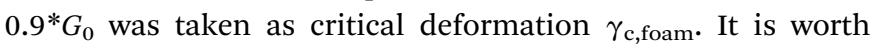
noting that this critical deformation at which non-linear deformation sets in is well below the yield point $\gamma_{\mathrm{y}}$ at which bubbles start to flow past each other. This yielding occurs at the stress or deformation amplitude at which $G^{\prime}=G^{\prime \prime}{ }^{7}$

\section{Results and discussion}

\subsection{Variation of ionic strength}

3.1.1 Foam and solution properties. In Table 1 the values of the surface tension measured after 30 minutes surface aging, the mean Sauter radii of the foam bubbles and the range of gas volume fractions where foam rheology measurements took place are shown. The surface tension does not significantly change with the addition of $\mathrm{NaCl}$ to the protein solutions. The initial mean bubble size decreases when adding $50 \mathrm{mM}$ salt but does not change upon further addition of $\mathrm{NaCl}$.

3.1.2 Oscillatory shear measurements. In Fig. 2 oscillatory shear stress amplitude sweeps are shown for the BLG foams with different ionic strengths at similar gas volume fraction $\phi$ $=89 \%$. For all foams $G^{\prime}$ and $G^{\prime \prime}$ stay nearly constant at low stresses in the linear viscoelastic regime. $G^{\prime}$ is always much higher than $G^{\prime \prime}$ and when $G^{\prime}$ increases, $G^{\prime \prime}$ also increases. All $G^{\prime}$ curves (Fig. 2a) show a decrease before they cross the $G^{\prime \prime}$ curve (intersections marked with crosses) with a negative slope increasing with increasing ionic strength. In the same stress amplitude range the $G^{\prime \prime}$ values (Fig. 2b) also show interesting behavior. For the foams without salt and with $10 \mathrm{mM} \mathrm{NaCl}$ the curves go through a local minimum and a subsequent local maximum just before crossing the $G^{\prime}$ curve. For higher ionic strengths the minimum in $G^{\prime \prime}$ essentially vanishes and the curves just exhibit a pronounced maximum which is shifted to higher stress amplitude values with increasing ionic strength. Such peaks have already been found for surfactant foams with gas volume fractions higher than $74 \% .^{30}$ The foams with lower gas volume fractions did not show this maximum which was
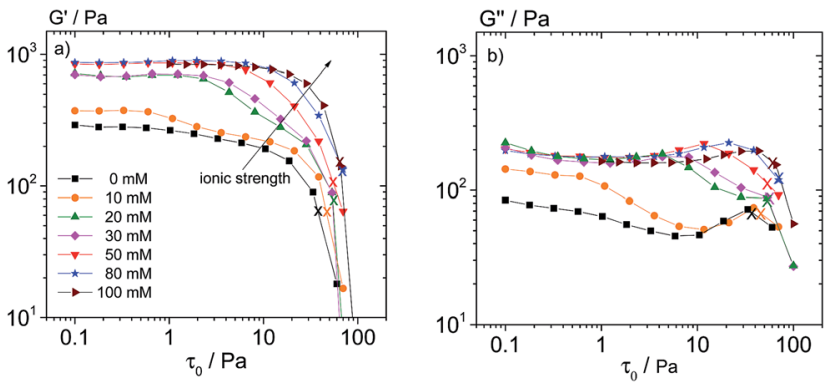

Fig. 2 Oscillatory shear experiments at a fixed frequency $f=1 \mathrm{~Hz}$ but varying stress amplitude for BLG foams $(\phi=89 \%)$ at different ionic strengths of $\mathrm{NaCl}$.

explained by the occurrence of plastic deformation prior to yielding. Other studies ${ }^{31,32}$ consider the point, where dissipation is maximal, as the transition point from elastic to viscous behavior, hence as the yield point. The simultaneous decrease of $G^{\prime}$ and $G^{\prime \prime}$ for salt concentrations up to $30 \mathrm{mM}$ indicates a gradual structure break down. Video recordings of sheared foams reveal that the bubbles start to slide past each other, i.e. the foam yields, when the maximum in $G^{\prime \prime}$ or the crossover of $G^{\prime}$ and $G^{\prime \prime}$ is reached as also reported in earlier studies. ${ }^{31,32}$ Similar behavior has been reported for whey protein isolate foams ${ }^{7}$ and was explained as follows: in several studies BLG has been found to form aggregates in thin liquid films that are able to "glue" the surfaces together. ${ }^{33,34}$ The simultaneous decrease of both moduli was interpreted as a gradual destruction of the intralamellar protein networks before the bubbles start to move past each other. But there is no direct experimental proof for this intralamellar network and a structural break down might also occur within the interface where the proteins form aggregated networks due to dominant attractive interactions. This network structure seems to be fully developed at high ionic strengths $(80 \mathrm{mM}, 100 \mathrm{mM})$ and then provides a uniform rigid surface layer stabilizing the foam and leading to a sharp decay of $G^{\prime}$ and $G^{\prime \prime}$ and a well defined yield point. At lower ionic strength the moduli decay simultaneously in a broad range of stress amplitude values between the onset of non-linear deformation and final yielding. The extended range between the LVE regime and the yield point is attributed to a gradual breakdown of the nonuniform, imperfect network structure within the interface (and/or across the lamellae) supposed to be present at lower ionic strength when attractive interactions are partly balanced by electrostatic repulsion.

Table 1 Surface tension of the protein solutions after 30 minutes surface aging, bubble radii and gas volume fractions in the range of foam ages where the rheological measurements took place. The maximum error is the standard deviation of three measurements at a given ionic strength

\begin{tabular}{llrrrrrr}
\hline Ionic strength $/ \mathrm{mM}$ & 0 & 10 & 20 & 30 & 50 & 80 & 100 \\
\hline$\sigma / \mathrm{mN} \mathrm{m}^{-1}$ & 51.5 & 50 & 49.5 & 49.6 & 50.1 & 49.5 & 49.9 \\
$r_{32} / \mu \mathrm{m}$ & $110-154$ & 114 & 121 & 124 & $78-175$ & $80-140$ & $77-145$ \\
$\phi / \%$ & $87-96$ & 89 & 89 & 89 & $86-93$ & $85-93$ & $84-91$
\end{tabular}



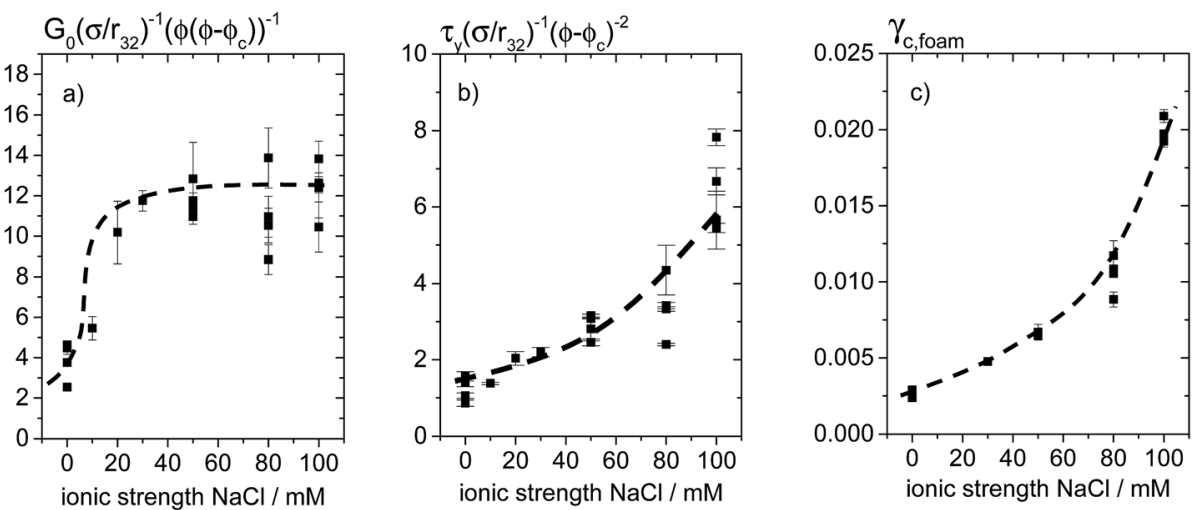

Fig. 3 (a) Storage moduli normalized by Laplace pressure $\left(\sigma / r_{32}\right)$ and $\phi\left(\phi-\phi_{c}\right)$, (b) yield stresses normalized by Laplace pressure and $\left(\phi-\phi_{\mathrm{c}}\right)^{2}$, and (c) critical deformation of BLG foams. All rheological quantities are measured at various gas volume fractions (see Table 1 ) and different ionic strengths.

3.1.3 Yield stress and storage modulus of the foams. Fig. 3 shows the yield stress values and the storage moduli normalized by Laplace pressure $\sigma / r_{32}$ and $\left(\phi-\phi_{\mathrm{c}}\right)^{2}$ or $\phi\left(\phi-\phi_{\mathrm{c}}\right)$, respectively and the critical deformation of the BLG foams for different $\mathrm{NaCl}$ concentrations. The normalization is derived from eqn (1) and (2) and results in a collapse of data taken at different gas volume fractions $\phi$ to a single master curve. The normalized storage moduli increase sharply and then levels off to a constant value of about 12 at an ionic strength of $30 \mathrm{mM}$. At such high salt concentrations adsorption of the proteins at the interface is enhanced due to a reduced electrostatic repulsion. Also a change to a compact folded shape that allows closer packing at the interface and increased lateral attraction due to counterion screening may occur. ${ }^{35}$ Accordingly, these closer packing of proteins increases the stress needed to deform the bubbles which corresponds to the measured storage modulus. Additionally, the protein aggregates that might occur more frequently at higher ionic strength could improve network formation thus further adding a mechanical strength. These effects are only observed up to $20 \mathrm{mM} \mathrm{NaCl}$, higher ionic strength does not lead to further increase of the normalized $G_{0}$ values. In contrast, the normalized yield stress and critical deformation increase monotonically with increasing ionic strength.

3.1.4 Surface rheology. Fig. 4a shows the elastic moduli of the surfaces in dilation $\left(E^{\prime}\right)$ and in shear $\left(G_{\mathrm{i}}^{\prime}\right)$. Both quantities show the same trend when increasing the ionic strength. Adding $10 \mathrm{mM} \mathrm{NaCl}$ leads to the increase of $E^{\prime}$ and $G_{\mathrm{i}}^{\prime}$ but further addition of salt does not have any effect. The increase of attractive interactions probably causes the higher $E^{\prime}$ and $G_{\mathrm{i}}^{\prime}$ values after addition of the salt. In Fig. $4 \mathrm{~b}$ and $\mathrm{c}$ the critical shear stress $\tau_{c \text {,surface }}$ and deformation $\gamma_{c \text {,surface }}$ of the surface layer are plotted versus the ionic strength. In contrast to the surface elastic moduli, $\tau_{\text {c,surface }}$ increases monotonically, indicating that a higher stress is required to destroy the interfacial layer structure formed at higher ionic strength. Finally, $\gamma_{\mathrm{c} \text {,surface }}$ $\approx \tau_{\mathrm{c} \text {,surface }} / G_{\mathrm{i}}^{\prime}$ increases monotonically with increasing ionic strength. Hence, the stress needed to deform the protein structure at the interface is not affected by ionic strength higher than $10 \mathrm{mM}$ but the stress and deformation that are needed to break the structure increase continuously with ionic strength in the range investigated here.
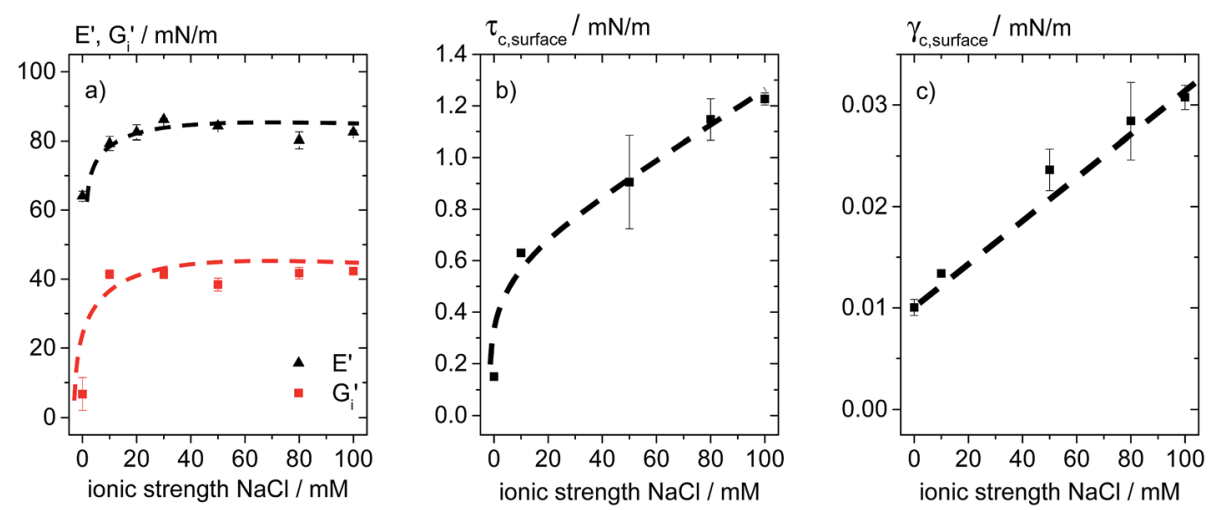

Fig. 4 (a) Surface elastic modulus in dilation $\left(E^{\prime}\right)$ and shear $\left(G_{i}^{\prime}\right)$, (b) critical shear stress $\tau_{c, \text { surface, }}$ and (c) critical deformation $\gamma_{c, \text { surface }}$ of the surface layer depending on the ionic strength for $1 \%$ BLG solutions. 
Table 2 Surface tension of the protein solutions with different kinds of salt after 30 minutes surface aging. Bubble radii and gas volume fractions were determined at various times in the range of foam ages where the rheological measurements took place. In all cases the salt concentration was chosen as $50 \mathrm{mM}$. The standard deviation of three measurements performed at a constant foam age and for a given kind of salt was calculated. The maximum standard deviation obtained from data for different foam ages and kinds of salt is listed here as maximum error

\begin{tabular}{lllllrr}
\hline & $\mathrm{NH}_{4} \mathrm{Cl}$ & $\mathrm{KCl}$ & $\mathrm{LiCl}$ & $\mathrm{CaCl}_{2}$ & $\mathrm{NdCl}_{3}$ & $\mathrm{Max}_{\text {error} / \%}$ \\
\hline$\sigma / \mathrm{mN} \mathrm{m}^{-1}$ & 46.6 & 48.7 & 47.5 & 47.1 & 48.7 & 2 \\
$r_{32} / \mu \mathrm{m}$ & $93-183$ & $84-175$ & $93-145$ & $105-278$ & $220-270$ & 10 \\
$\phi / \%$ & $86-92$ & $82-90$ & $84-90$ & $84-91$ & $92-95$ & 1 \\
$\phi_{\mathrm{c}}$ & 71.5 & 71.3 & 70.7 & 73.0 & 84.9 & 4
\end{tabular}

\subsection{Variation of the kind and the valency of the cation}

3.2.1 Foam and solution properties. Table 2 shows the characteristic properties of the solutions and foams prepared with $50 \mathrm{mM}$ salt of different types and valencies. The surface tension is not significantly affected by the kind of added salt, whereas the initial bubble radii and the bubble size distribution increase with the valency of the cation. Especially when $50 \mathrm{mM}$ $\mathrm{NdCl}_{3}$ were added, the foams possess comparably big bubbles with a broad size distribution as reflected by the high $\phi_{\mathrm{c}}$ value. $^{7}$ This is most likely due to the occurrence of aggregated proteins as a consequence of the strongly suppressed electrostatic repulsion in the presence of trivalent ions. More and/or bigger protein clusters result in lower affinity of the proteins to adsorb at the interface. Hence, not all arising bubbles can be immediately stabilized what leads to an overall increase of the bubble size and a broader distribution.

3.2.2 Yield stress and storage modulus of the foams. Fig. 5a-c show the reduced storage moduli, reduced yield stresses and $\gamma_{c \text {,foam }}$ data, respectively for the different salts added. No difference is observed for the foams made from solutions including different monovalent salts. The addition of the divalent salt $\mathrm{CaCl}_{2}$ leads to lower values in reduced $G_{0}$ but to

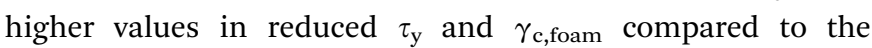
monovalent salts. In particular, for the latter the difference is very pronounced. Each ion behaves differently when coming into contact with the protein. It is a balance between binding to the protein and preferential hydration (exclusion of the salt from the protein surface) of the protein. ${ }^{36,37} \mathrm{Ca}^{2+}$ is known to bind very strongly to BLG which could lead to conformational changes of the protein resulting in an increase of the hydrophobic surface area leading to stronger protein-protein interactions. Also, the formation of ionic bridges cross-linking the protein molecules is likely to occur. ${ }^{38,39}$ The results for the foams made from BLG solutions with $50 \mathrm{mM} \mathrm{NdCl}_{3}$ also differ strongly from those obtained for foams including monovalent ions. For both, the reduced $G_{0}$ and $\tau_{\mathrm{y}}$, we have found very high values, whereas $\gamma_{\mathrm{c} \text {,surface }}$ is not significantly higher than for the monovalent ions. The reason for the high $\tau_{\mathrm{y}}$ and $G_{0}$ values is again attributed to aggregation of proteins resulting from their low solubility at this high ionic strength. This is supported by the turbidity of the solutions observed after adding the salt. The mechanism that causes these high values of the rheological parameters is presumably again the formation of a structure across the lamellae. The reduced $\tau_{\mathrm{y}}$ values additionally vary with gas volume fraction $\phi$. In fact, the $\phi$-dependent $\tau_{\mathrm{y}}$ for these foams is lower than that predicted by eqn (2). We assume that the network structure is not destroyed as long as $\tau<\tau_{\mathrm{y}}$ as it was the case for the foams investigated in ref. 7 , but additionally contributes to the high yield stress. At lower $\phi$ and therefore thicker lamellae the protein network spanning the lamellae is expected to have more influence and hence causes high yield stress values leading to the unexpected weak variation of $\tau_{\mathrm{y}}$ with $\phi$. This phenomenon does not show up in the critical deformation of the foams. Finally, the different effects of divalent $\mathrm{Ca}^{2+}$ and trivalent $\mathrm{Nd}^{3+}$ on foam rheology clearly demonstrate that the corresponding protein structure and packing is strongly affected by the type and valency of the added ions.
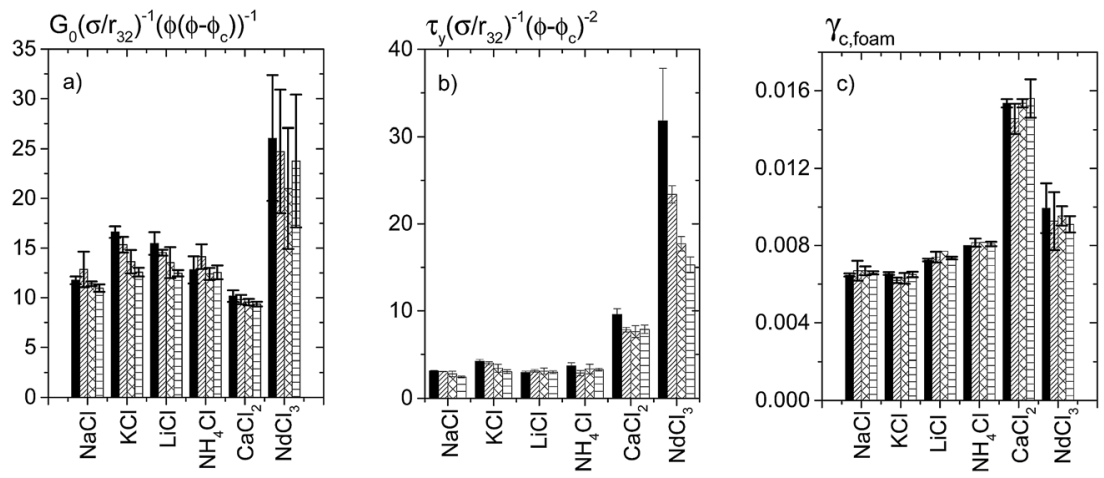

Fig. 5 (a) Reduced storage moduli, (b) reduced yield stresses and (c) critical deformation for foams made from $1 \%$ BLG dissolved in an aqueous $50 \mathrm{mM}$ salt solution measured at different gas volume fractions $\phi$ (see Table 2). For each salt $\phi$ increases from left to right. 

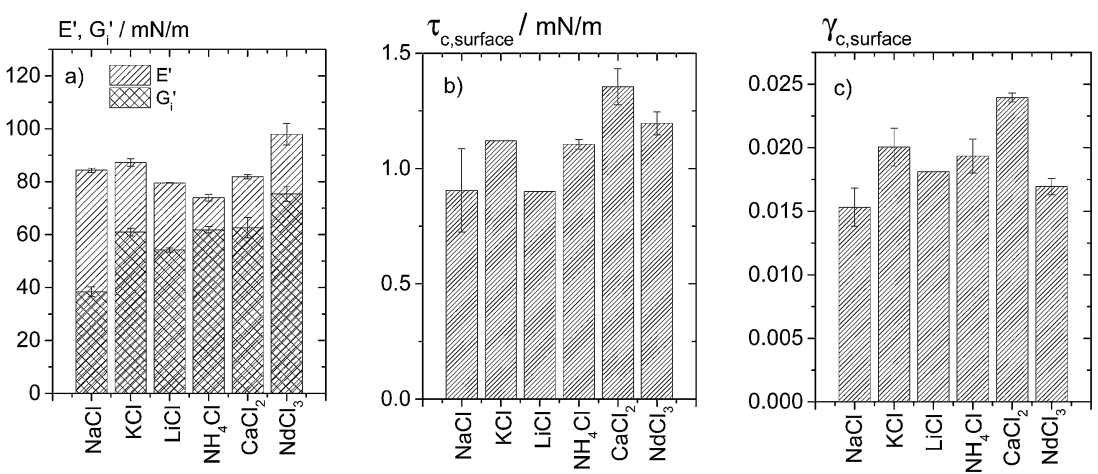

Fig. 6 (a) Surface elastic modulus in dilation $\left(E^{\prime}\right)$ and shear $\left(G_{i}^{\prime}\right)$, (b) critical shear stress $\tau_{c, \text { surface }}$ and (c) critical deformation $\gamma_{c, \text { surface }}$ of the surface layer for $1 \%$ BLG solutions containing $50 \mathrm{mM}$ salt of different types and valency.

3.2.3 Surface rheology. In Fig. 6a-c the surface moduli $E^{\prime}$ and $G_{\mathrm{i}}^{\prime}$, the critical deformation of the surface layer $\gamma_{\mathrm{c} \text {,surface }}$ and the critical stress of the surface layer $\tau_{\mathrm{c} \text {,surface }}$ are shown for the protein solutions containing different salts. The results show similarities to those found for the foams (Section 3.2.2). Monovalent ions affect $E^{\prime}, G_{\mathrm{i}}^{\prime}, \gamma_{\mathrm{c} \text {,surface }}$ and $\tau_{\mathrm{c} \text {,surface }}$ in a similar way. $\mathrm{CaCl}_{2}$ increases $\gamma_{\mathrm{c} \text {,surface }}$ and $\tau_{\mathrm{c} \text {,surface }}$ but not the surface elastic moduli. The stronger binding of $\mathrm{Ca}^{2+}$, as described in Section 3.2.2 may also explain why the critical stress and deformation needed to break the protein structure is higher with $\mathrm{Ca}^{2+}$ than for other ions. The solutions containing $\mathrm{NdCl}_{3}$ show the highest $E^{\prime}$ and $G_{\mathrm{i}}^{\prime}$ values but low $\gamma_{\mathrm{c} \text {,surface }}$ values similar to the monovalent case and $\tau_{\mathrm{c}, \text { surface }}$ is in between the values for the monovalent ions and $\mathrm{Ca}^{2+}$. In general, the effect of di- and trivalent ions on interfacial rheology is much less pronounced than on foam rheology. This strongly suggests that foam rheology in these cases is strongly determined by the structure formation across the lamellae.

\subsection{Variation of $\mathrm{pH}$}

3.3.1 Foam and solution properties. Table 3 shows the characteristic properties of the BLG foams and solutions at different $\mathrm{pH}$. The initial average bubble sizes and bubble size distributions $\left(\phi_{\mathrm{c}}=71.5 \pm 1.9\right.$ for all foams) are practically independent of $\mathrm{pH}$. The surface tension varies with a minimum at pH 5 as already found in ref. 24 and 39.

3.3.2 Oscillatory shear stress amplitude sweeps. In Fig. 7 oscillatory shear stress amplitude sweeps for foams at $\mathrm{pH}$ values between 3 and 8 are shown. Interestingly, the curves possess different shapes at different $\mathrm{pH}$. At and below the IEP $(\approx \mathrm{pH} 5)$
$G^{\prime}$ and $G^{\prime \prime}$ are almost constant before crossing (at pH 3 the foams are very unstable and therefore the moduli slightly decrease). The curves obtained at $\mathrm{pH}$ above the IEP can be divided into four regimes. After a short linear viscoelastic regime, both moduli decrease simultaneously, then $G^{\prime \prime}$ increases again before the moduli cross over and finally both decrease again. The simultaneous decrease of $G^{\prime}$ and $G^{\prime \prime}$ indicates a gradual structural break down as described in Section 3.1.2.

3.3.3 Yield stress and storage modulus of the foams. In Fig. 8 the plateau moduli and yield stresses of BLG foams at various gas volume fractions normalized by the Laplace pressure as well as the critical deformation are shown depending on the $\mathrm{pH}$. Around the isoelectric point the foams exhibit the maximum yield stress, elastic modulus and critical deformation. Low $\mathrm{pH}$, especially $\mathrm{pH} 3$, leads to very unstable foams that possess very low elasticity and yield stress whereas foams at high $\mathrm{pH}$ are fairly stable. This behavior has already been reported in ref. 39 and was explained by conformational changes of the protein structure at different $\mathrm{pH}$. Also, different electrostatic interactions between the proteins due to the change in their net charge across the IEP occur. At pH 5 the net charge vanishes which was shown to lead to thick disordered protein layers at the surface. The proteins also tend to aggregate at the isoelectric point. Once trapped in a foam lamella these protein clusters presumably support the network formation across two adjacent surfaces and give additional mechanical strength to the foam structure resulting in such high values for storage modulus and yield stress. In ref. 7 the proposed network formation in whey protein isolate foam lamellae was found to

Table 3 Surface tension of the protein solutions at various $\mathrm{pH}$ after 30 minutes surface aging, bubble radii and gas volume fractions in the range of foam ages where the rheological measurements took place. The maximum error is the maximum standard deviation of three measurements at a given $\mathrm{pH}$

\begin{tabular}{llllllll}
\hline $\mathrm{pH}$ & 3 & 4 & 5 & 6 & 6.8 & 8 & 9 \\
\hline$\sigma / \mathrm{mN} \mathrm{m}^{-1}$ & 47.6 & 48.3 & 45.5 & 48.9 & 51.5 & 52.2 & 52.0 \\
$r_{32} / \mu \mathrm{m}$ & $104-109$ & 118 & $93.8-145$ & 102 & $110-154$ & 103 & $106-175$ \\
$\Phi / \%$ & $86-88$ & 88 & $84-91$ & 89 & $87-96$ & 88 & $87-94$
\end{tabular}



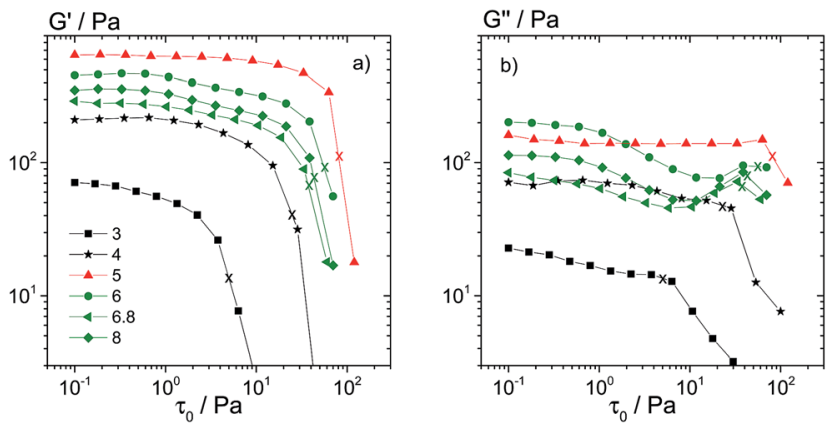

Fig. 7 Oscillatory shear measurements with varying stress amplitude of BLG foams at different pH: (a) storage modulus $G^{\prime}$ and (b) loss modulus $G^{\prime \prime}$ versus the stress amplitude at a fixed frequency $f=1 \mathrm{~Hz}$. The intersection of $G^{\prime}$ and $G^{\prime \prime}$ is marked with crosses.

increase $G_{0}$ but did not affect the yield stress. From oscillatory amplitude sweeps it could be concluded that the whey protein network is destroyed at $\tau<\tau_{y}$, as it is the case for every $\mathrm{pH} \neq 5$. This suggests the existence of weak, non-uniform networks gradually destroyed as the stress amplitude increases. At $\mathrm{pH} 5$ the destruction of the protein network seems to go along with yielding of the foam indicating a strong uniformly collapsing structure. Above $\mathrm{pH} 6.8$ all three foam rheological parameters do not significantly depend on $\mathrm{pH}$.

3.3.4 Surface rheology. Fig. 9a shows the $\mathrm{pH}$ dependence of the surface elastic moduli in shear and dilation for the $1 \%$ BLG solutions. Both moduli increase up to $\mathrm{pH} 6$ followed by a local minimum around $\mathrm{pH}$ 7. The minimum probably arises because this is the natural $\mathrm{pH}$ of the solution and no ions are added to adjust the $\mathrm{pH}$. The maximum change in the ionic strength due to adjusting $\mathrm{pH}$ is approximately $10 \mathrm{mM}$ and according to Fig. 4 a this corresponds to a drastic increase in $G_{\mathrm{i}}^{\prime}$ as well as $E^{\prime}$. Against expectation, the surface moduli do not show a maximum around the IEP as it has been reported for various proteins including BLG in several studies of interfacial shear ${ }^{41-44}$ and dilational properties. ${ }^{24,40,45}$ But it should be noted that the protein concentration used in those studies is much lower (at least 5-10 times) than the concentration used here which was chosen to be that high to meet the conditions used for foam preparation and foam rheology measurement. Burgess et al. ${ }^{35}$ have also worked with high protein concentrations of BSA and $\mathrm{HI}_{\mathrm{gG}}$ similar to the concentrations used in this study and measured surface shear elasticities as a function of $\mathrm{pH}$. Surprisingly, they found a minimum in $G_{\mathrm{i}}^{\prime}$ at the IEP and explained this phenomenon by low protein-protein interactions due to a compact shape of the proteins. This explanation is in disagreement with the other studies mentioned above which conclude a strong attraction among proteins at the IEP. They attributed the attractive interactions to close and effective contacts among proteins because of the low net charge. ${ }^{24,35} \mathrm{We}$ assume that the difference in the reported results arises mainly from different protein concentrations. Exceeding a critical protein concentration results in the formation of multilayers at the surface. Wüstneck ${ }^{46}$ measured surface elastic properties of gelatin layers and found the elastic modulus to decrease at a certain concentration that he attributed to the onset of multilayer formation. Also, the formation of protein aggregates, which becomes more significant as the protein concentration increases, can lead to a decrease in the surface elasticity as we discussed extensively in a previous study. ${ }^{7}$ The reason for the lower values at $\mathrm{pH} 3$ compared to the values at $\mathrm{pH} 6.8$ despite the same distance to the isoelectric point is differences in the protein structure and hydrophobicity as already discussed in ref. 24 and 40.

\subsection{Correlation between interfacial and foam rheology}

In Fig. 10 the normalized storage moduli of all foams investigated in this study are plotted versus the surface elastic moduli $G_{\mathrm{i}}^{\prime}$ and $E^{\prime}$. Additionally, data points for whey protein isolate foams $(0.1 \%$ and $1 \%)$ and $3 \%$ casein foams (data taken from ref. 7) are included. A clear correlation is observed between the normalized foam moduli and surface moduli of the corresponding protein solutions except for the 1\% BLG solutions at pH 5 and with $\mathrm{NdCl}_{3}$ as well as the $1 \%$ WPI solution. The relationship between $G_{0}$ and $G_{\mathrm{i}}^{\prime}$ is obviously linear whereas our data suggest a stronger quadratic or cubed dependence of $G_{0}$ on $E^{\prime}$. These findings directly demonstrate that surface elasticity is
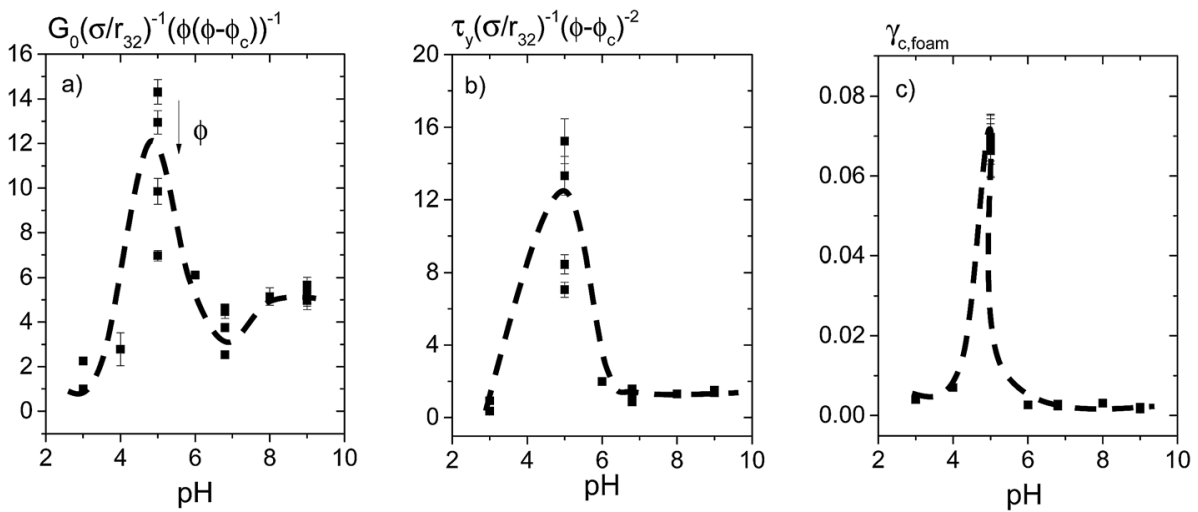

Fig. 8 (a) Storage moduli normalized by Laplace pressure $\left(\sigma / r_{32}\right)$ and $\phi\left(\phi-\phi_{\mathrm{c}}\right)$, (b) yield stresses normalized by Laplace pressure and $\left(\phi-\phi_{\mathrm{c}}\right)^{2}$ and (c) critical deformation of BLG foams. All rheological quantities are measured at various gas volume fractions (see Table 3 ) and different $\mathrm{pH}$. 

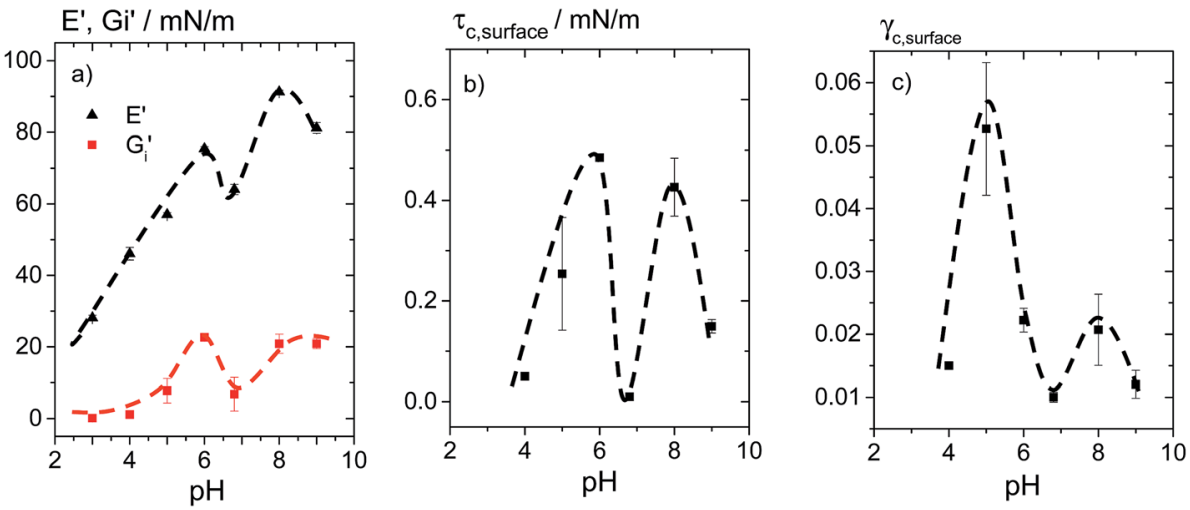

Fig. 9 (a) Surface elastic modulus in dilation $\left(E^{\prime}\right)$ and shear $\left(G_{i}^{\prime}\right)$, (b) critical shear stress $\tau_{c, \text { surface }}$ and (c) critical deformation $\gamma_{c, \text { surface }}$ of the surface layer for $1 \%$ BLG solutions at different $\mathrm{pH}$.
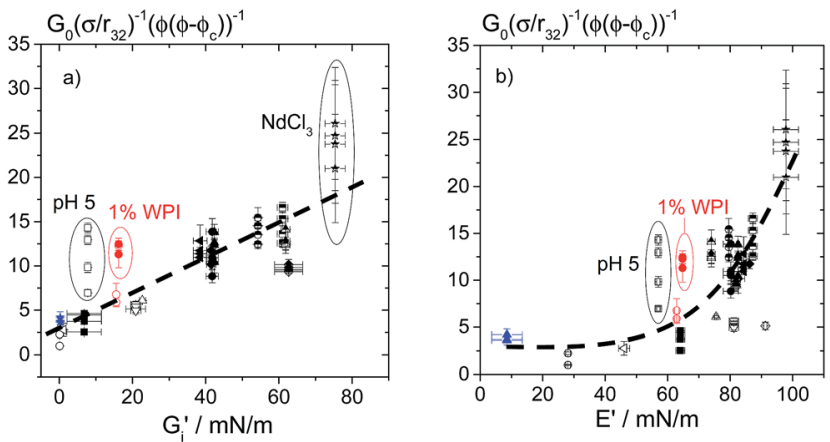

Fig. 10 Storage moduli normalized by Laplace pressure $\left(\sigma / r_{32}\right)$ and $\phi(\phi$ $-\phi_{c}$ ) versus (a) surface shear elastic modulus $G_{i}^{\prime}$ and (b) surface dilational elastic modulus $E^{\prime} \mathrm{CpH} 3, \triangle \mathrm{pH} 4, \square \mathrm{pH} 5, \triangle \mathrm{pH} 6, \diamond \mathrm{pH} 8, \nabla \mathrm{pH}$ 9, $\mathrm{pH} 6.8$ and NaCl: $0 \mathrm{mM}, \diamond 10 \mathrm{mM}, \Delta 50 \mathrm{mM},-80 \mathrm{mM}, \Delta 100 \mathrm{mM}, 50$ $\mathrm{mM} \nabla \mathrm{KCl}, \Delta \mathrm{NH}_{4} \mathrm{Cl}$, $\bullet \mathrm{LiCl}, \diamond \mathrm{CaCl}_{2}, \star \mathrm{NdCl}_{3} \bigcirc 0.1 \% \mathrm{WPI}, 1 \% \mathrm{WPI}, \Delta 3 \%$ casein.

another important parameter controlling foam elasticity besides the Laplace pressure inside the bubbles, gas volume fraction and bubble size distribution. Accordingly, the prefactor $a$ in eqn (1) is solely determined by the surface elastic moduli $G_{\mathrm{i}}^{\prime}$ or $E^{\prime}$. For the three cases where deviations from the simple correlations between foam and surface elasticity are observed, protein aggregation and structure or network formation across foam lamellae are supposed to be decisive for foam elasticity as already discussed in detail in Section 3.2 and 3.3, as well as ref. 7.

Fig. $11 \mathrm{a}$ and $\mathrm{b}$ display the reduced yield stresses of all investigated BLG foams as a function of critical stress $\tau_{\mathrm{c} \text {,surface }}$ or $\gamma_{c \text {,surface }}$ characterizing the onset of non-linear response of the corresponding protein solutions in interfacial shear rheology experiments. The relationship between the normalized foam yield stress and these characteristic surface rheological parameters can be approximated by a linear correlation as the simplest approach. This demonstrates that the parameter $k$ in eqn (2) is determined by surface rheological features of the corresponding BLG solutions. But again the foams made at $\mathrm{pH}$ 5 and those made from the BLG solutions including $50 \mathrm{mM}$ $\mathrm{NdCl}_{3}$ clearly deviate from this simple correlation and again we conclude that this is due to a structure or network formation across foam lamellae as discussed above which also dominates the yielding of the foam. Finally, Fig. 11c shows the correlation between the critical deformation $\gamma_{c}$,foam characterizing the
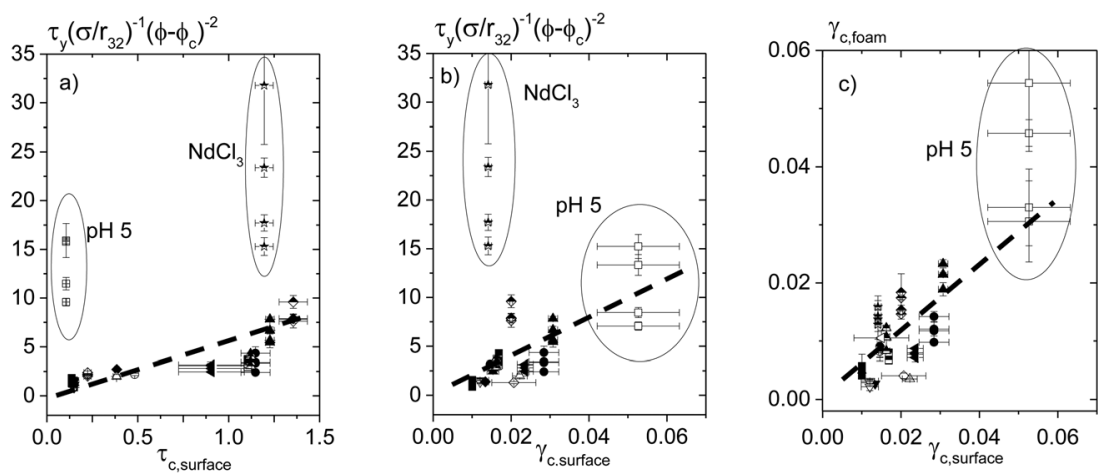

Fig. 11 Yield stresses normalized by Laplace pressure and $\left(\phi-\phi_{c}\right)^{2}$ versus (a) critical shear deformation of the surface $\tau_{c, \text { surface, }}(\mathrm{b}) \mathrm{critical}$ shear deformation of the surface $\gamma_{c, \text { surface, }}$ and (c) critical deformation of the foams $\gamma_{c \text {,foam }}$ versus critical deformation of the surface $\gamma_{c, \text { surface. }}$. $\mathrm{pH} 3, \triangle$

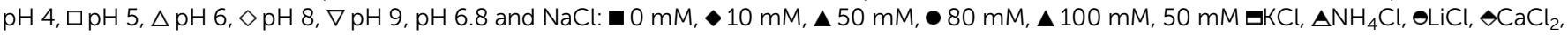
$\star \mathrm{NdCl}_{3}$. 
onset of non-linear response during oscillatory shear of the foams and the critical deformation $\gamma_{\mathrm{c} \text {,surface }}$ obtained in oscillatory surface shear experiments. Again, a clear correlation between characteristic foam and surface rheological properties is found including all investigated BLG systems except those at $\mathrm{pH}$ 5. This once more confirms that foam rheology is tightly related to surface rheological properties of the corresponding protein solutions. Moreover, the deviation of the data for the BLG systems at $\mathrm{pH} 5$ from this correlation and the strong dependence of $\gamma_{c \text {,surface }}$ on gas volume fraction further supports the conclusion that in this case foam rheology is dominated by the structure across the foam lamellae instead of the opposing protein surface layers alone.

\section{Conclusions}

We have investigated the correlation between the rheological behavior of BLG foams and the surface shear and dilational viscoelastic properties of corresponding protein solutions. Foam rheology is characterized here in terms of the frequency independent storage modulus $G_{0}$ reflecting the elasticity of the foam "at rest" and the apparent yield stress $\tau_{\mathrm{y}}$ determining the onset of flow, i.e. the stress at which bubbles start to slide past each other. Moreover, the transition from linear to non-linear response was characterized using stress amplitude sweep oscillatory shear experiments. From these experiments the critical strain $\gamma_{c}$ defining the cessation of linear viscoelastic response was extracted. These foam rheological quantities were determined at gas volume fractions between $82 \%$ and $96 \%$. The surface viscoelasticity was characterized in shear and dilation. The corresponding shear $G_{\mathrm{i}}^{\prime}$ and dilational $E^{\prime}$ moduli as well as the critical strain $\gamma_{c}$ and stress $\tau_{c}$ marking the onset of nonlinear response in oscillatory surface shear experiments were determined at a fixed frequency. Solution $\mathrm{pH}$ as well as concentration, type and valency of the added salt has been varied systematically thus varying foam rheology and surface viscoelasticity in a wide range.

Since protein conformation, solubility and aggregation in the bulk and at the surface may strongly change with protein concentration surface viscoelastic properties were determined at the same protein concentration as used for foam preparation.

Foam as well as interfacial moduli $G_{0}, G_{\mathrm{i}}^{\prime}$ and $E^{\prime}$, respectively, strongly increases upon addition of salt to the protein solution but levels off at an ionic strength of about 20-30 mM NaCl. The quantities $\tau_{\mathrm{y}}, \gamma_{\mathrm{c} \text {,foam }}, \tau_{\mathrm{c} \text {,surface }}$, and $\gamma_{\mathrm{c} \text {,surface }}$ characterizing the transition from linear to non-linear response increase monotonically with increasing ionic strength. A characteristic change from a gradual decrease of $G^{\prime}$ with increasing stress amplitude in oscillatory shear experiments to a sharp decrease at a higher critical stress is found when more and more salt is added. This indicates the formation of a stronger and more uniform structure of the foam and the interfacial protein layers as the attractive interactions among proteins become dominant.

The type and valency of the added salt has little effect on the surface viscoelastic properties of the protein solutions. But foam rheology drastically changes when divalent $\left(\mathrm{Ca}^{2+}\right)$ or trivalent ions $\left(\mathrm{Nd}^{3+}\right)$ are added. Adding $\mathrm{Nd}^{3+}$ results in a drastic increase in $G_{0}$ and $\tau_{\mathrm{y}}$ but also in an anomalously weak variation of $\tau_{\mathrm{y}}$ with gas volume fraction not captured by eqn (2). In contrast, addition of $\mathrm{Ca}^{2+}$ mainly shows up in a strong increase of $\gamma_{c \text {,foam. }}$. These findings indicate the formation of an aggregated protein network structure across foam lamellae, which then determines the foam properties but does not show up in interfacial viscoelasticity. The different effects of $\mathrm{Nd}^{3+}$ and $\mathrm{Ca}^{2+}$ suggest that different structures are formed within the lamellae.

Variation of $\mathrm{pH}$ has little effect on foam rheological parameters $G_{0}, \tau_{\mathrm{y}}$ and $\gamma_{\mathrm{c} \text {,foam }}$ except at $\mathrm{pH} 5$ which is the isoelectric point. At this point all these quantities exhibit distinct maxima and again $\tau_{\mathrm{y}}$ and $G_{0}$ show an unusually weak dependence on the volume fraction not captured by the scaling laws (eqn (1) and (2)) confirmed by various previous studies. This again indicates the formation of a network structure of aggregated protein molecules across the lamellae and this is further supported by the non-monotonic variation of surface viscoelastic parameters not showing similar strong and distinct maxima at pH 5.

Finally, a unique correlation between foam rheological properties and surface viscoelasticity of corresponding BLG solutions could be established using all the collected data mentioned above. The reduced foam storage modulus increases monotonically with $G_{\mathrm{i}}^{\prime}$ and $E^{\prime}$ except for the foams made at pH 5 and in the presence of the trivalent salt. Moreover, the correlation between the reduced apparent yield stress and $\tau_{\mathrm{c} \text {,surface }}$ or $\gamma_{c, \text { surface }}$ is well approximated by a linear relationship and $\gamma_{c \text {,foam }}$ is proportional to $\gamma_{c \text {,surface }}$ within experimental uncertainty. Once more, the foam rheological parameters obtained at pH 5 and when $\mathrm{Nd}^{3+}$ is added are significantly higher than expected from these simple correlations.

In summary, we conclude that the widely accepted physical models predicting foam modulus and yield stress from the Laplace pressure within the gas bubbles and the gas volume fraction do not fully capture the physics of these phenomena. The pre-factors $a$ and $k$ in eqn (1) and (2) are not just numerical constants on the order of one but are found to vary between $1<$ $a$ and $k<30$. A unique correlation between foam rheological properties and surface viscoelastic parameters is found except in cases where attractive interactions among proteins are dominant and are supposed to be strong enough to form a network structure across foam lamellae.

\section{Acknowledgements}

The authors would like to thank the group of U. Kulozik (Technische Universität München, Germany) for the supply of high quality $\beta$-lactoglobulin and the groups of $\mathrm{W}$. Peukert (Universität Erlangen-Nürnberg, Germany) and M. Wilhelm (Karlsruhe Institute of Technology, Germany) for access to measuring equipments. We acknowledge funding by the DFGAiF cluster project "Mesoscale modeling of the rheology of foamed food products” Wi 3138/10-1.

\section{References}

1 T. G. Mason, J. Bibette and D. A. Weitz, Phys. Rev. Lett., 1995, 75, 2051-2054. 
2 T. G. Mason, J. Bibette and D. A. Weitz, J. Colloid Interface Sci., 1996, 179, 439-448.

3 H. M. Princen, J. Colloid Interface Sci., 1986, 112, 427-437.

4 H. M. Princen and A. D. Kiss, J. Colloid Interface Sci., 1989, 128, 176-187.

5 S. Marze, A. Saint-Jalmes and D. Langevin, Colloids Surf., A, 2005, 263, 121-128.

6 M. Lexis and N. Willenbacher, Chem. Ing. Tech., 2013, 85, 1317-1323.

7 M. Lexis and N. Willenbacher, Colloids Surf., A, 2014, 459, 177-185.

8 N. Willenbacher and M. Lexis, in Foam Films and Foams: Fundamentals and Application, ed. R. Miller and L. Liggieri, Tayler and Francis, 2014, in preparation.

9 P. Cicuta and E. M. Terentjev, Eur. Phys. J. E, 2005, 16, 147158.

10 J. Krägel, S. Derkatch and R. Miller, Adv. Colloid Interface Sci., 2008, 144, 38-53.

11 A. Martin, M. Bos, M. Cohen Stuart and T. van Vliet, Langmuir, 2002, 18, 1238-1243.

12 S. Rouimi, C. Schorsch, C. Valentini and S. Vaslin, Food Hydrocolloids, 2005, 19, 467-478.

13 M. A. Bos, B. Dunnewind and T. van Vliet, Colloids Surf., B, 2003, 31, 95-105.

14 A. Martin, K. Grolle, M. Bos, M. Stuart and T. van Vliet, J. Colloid Interface Sci., 2002, 254, 175-183.

15 J. Maldonado-Valderrama, A. Martín-Rodriguez, M. J. GálvezRuiz, R. Miller, D. Langevin and M. A. Cabrerizo-Vílchez, Colloids Surf., A, 2008, 323, 116-122.

16 P. Wilde, Curr. Opin. Colloid Interface Sci., 2000, 5, 176-181.

17 H. M. Princen, J. Colloid Interface Sci., 1983, 91, 160-175.

18 S. Besson, G. Debrégeas, S. Cohen-Addad and R. Höhler, Phys. Rev. Lett., 2008, 101, 214504.

19 K. Krishan, A. Helal, R. Höhler and S. Cohen-Addad, Phys. Rev. E: Stat., Nonlinear, Soft Matter Phys., 2010, 82, 011405.

20 S. Costa, R. Höhler and S. Cohen-Addad, Soft Matter, 2012, 9, 1100.

21 N. Denkov, S. Tcholakova, K. Golemanov, K. Ananthapadmanabhan and A. Lips, Phys. Rev. Lett., 2008, 100, 138301.

22 N. D. Denkov, S. Tcholakova, K. Golemanov, K. P. Ananthpadmanabhan and A. Lips, Soft Matter, 2009, 5, 3389.

23 N. D. Denkov, V. Subramanian, D. Gurovich and A. Lips, Colloids Surf., A, 2005, 263, 129-145.
24 J. Davis, E. Foegeding and F. Hansen, Colloids Surf., B, 2004, $34,13-23$.

25 T. D. Dimitrova and F. Leal-Calderon, Adv. Colloid Interface Sci., 2004, 108-109, 49-61.

26 S. Arditty, V. Schmitt, F. Lequeux and F. Leal-Calderon, Eur. Phys. J. B, 2005, 44, 381-393.

27 S. Vandebril, A. Franck, G. G. Fuller, P. Moldenaers and J. Vermant, Rheol. Acta, 2010, 49, 131-144.

28 A. Saint-Jalmes, Y. Zhang and D. Langevin, Eur. Phys. J. E, 2004, 15, 53-60.

29 M. Durand and D. Langevin, Eur. Phys. J. E, 2002, 35-44.

30 A. Saint-Jalmes and D. J. Durian, J. Rheol., 1999, 43, 14111422.

31 S. Marze, R. M. Guillermic and A. Saint-Jalmes, Soft Matter, 2009, 5, 1937.

32 N. Jager-Lézer, J.-F. Tranchant, V. Alard, C. Vu, J.-L. Grossiord and P. C. Tchoreloff, Rheol. Acta, 1998, 37, 129-138.

33 B. Rullier, M. A. Axelos, D. Langevin and B. Novales, J. Colloid Interface Sci., 2009, 336, 750-755.

34 B. Rullier, B. Novales and M. A. Axelos, Colloids Surf., A, 2008, 330, 96-102.

35 D. J. Burgess and N. O. Sahin, J. Colloid Interface Sci., 1997, 189, 74-92.

36 R. A. Curtis, J. M. Prausnitz and H. Blanch, Biotechnol. Bioeng., 1998, 57, 11-21.

37 S. N. Timasheff and T. Arakawa, J. Cryst. Growth, 1988, 90, 39-46.

38 H. Zhu and S. Damodaran, J. Agric. Food Chem., 1994, 42, 856-862.

39 C. A. Zittle, E. S. DellaMonica, R. K. Rudd and J. H. Custer, J. Am. Chem. Soc., 1957, 79, 4661-4666.

40 K. Engelhardt, M. Lexis, G. Gochev, C. Konnerth, R. Miller, N. Willenbacher, W. Peukert and B. Braunschweig, Langmuir, 2013, 29, 11646-11655.

41 D. Graham and M. Phillips, J. Colloid Interface Sci., 1980, 76, 240-250.

42 S. Pezennec, F. Gauthier, C. Alonso, F. Graner, T. Croguennec, G. Brulé and A. Renault, Food Hydrocolloids, 2000, 14, 463-472.

43 S. H. Kim and J. E. Kinsella, J. Food Sci., 1985, 50, 1526-1530. 44 S. A. Roberts, I. W. Kellaway, K. M. G. Taylor, B. Warburton and K. Peters, Langmuir, 2005, 21, 7342-7348.

45 D. Graham and M. Phillips, J. Colloid Interface Sci., 1980, 76, 227-239.

46 R. Wüstneck, Colloid Polym. Sci., 1984, 262, 821-826. 